\title{
Is aneuploidy a defense mechanism to prevent maternity later in a woman's life
}

\author{
Marc-André Sirard
}

Published online: 9 December 2010

(C) Springer Science+Business Media, LLC 2010

Meiosis, the process by which half of the chromosomes of a parent are passed to the next generation, appeared on earth more than 2 billion years ago and is part of the reproduction process of all plants and animals [1]. A very similar process mitosis; by which a cell transmits a copy of all its chromosomes to 2 daughter cells, has been around for even longer and occurs millions of times a day in any animal

Aneuploidy is a condition in which cells have an abnormal number of chromosomes. This occurs mainly during meiosis and results from different types of problems in the complex apparatus joining and separating homologous chromosomes. This condition is particularly problematic in human embryos from older female patients. In the scientific literature as well as among the clinical community, aneuploidy is considered a fact of life. However, it is legitimate to ask why would the meiotic process be prone to mistakes after millions of years of evolution? And why would this process be error prone in only very few species? And more importantly, why does aneuploidy occur in humans at a much higher frequency in females of advancing age?

Aneuploidy has been first analysed through the perspective of a biological process that requires an exchange of fragments between the maternal and paternal chromosomes. This exchange process could affect some chromosomes to the point of mis-segregation at the anaphase-telophase stage. The segregation process is a well known and well

Capsule This editorial (text) explores the potential influence of evolution on the high rates of oocyte aneuploidy in humans.

M.-A. Sirard $(\bowtie)$

Chaire de Recherche du Canada en Génomique Animale, Centre de Recherche en Biologie de la Reproduction,

Pavillon des services, INAF, bureau 2732, Université Laval, Laval G1K 7P4 Quebec, Canada

e-mail: Marc-Andre.Sirard@fsaa.ulaval.ca studied in animal models and although they might explain what goes wrong if a given factor or protein is missing, they fail to justify why it is so prominent in human females after 40 years of age.

Obviously time could be a factor by itself. The meiotic process begins in the womb for female embryos and then stops midway until the oocyte is selected for ovulation. This holding period can last as long as 40 years or more before the rest of the division process resumes at ovulation. But why would there be such a difference between the ages of 30 and 40 when aneuploidy increases exponentially if the effect of time is linear?

Another simple explanation is the aging process. Indeed, as the body ages, ovaries, and the oocytes they contain, age as well. It follows that one could expect the same aging phenotype in oocyte as in somatic cells. A different explanation points to impaired oocyte selection as a result of either a less stringent process within fewer follicles instead of an increase in the relative amount of aneuploid oocytes with age [2]. But if we put aside the mechanism of how it happens, the question of why it happens remains unanswered.

A promising explanation that has not, to my knowledge, been explored is the likelihood that the ovary purposely induces aneuploidy in selected conditions. For this apparent sabotage to make any evolutionary sense however, it should benefit evolution in the long run. Is this a workable hypothesis?

Before exploring such, another phenomenon peculiar to humans should be considered: menopause. For this unusual phenotype, which does not happen as such in other mammals, at least two major explanations are still being debated. The first one claims that the ovary need not to store eggs for longer than the average survival age of females, which in humans used to be much shorter than nowadays and corresponded to the approximate time of menopause. The rationale underlying this first explanation 
rests on a simple saving of gametes. The second explanation refers to the potential advantage for offspring of being looked after by an available mother, if not a grandmother, in order to increase their chances of survival and reproduction [3]. Firstly, mothers who stop having children reduce their chances of dying during childbirth, which used to be quite common. Secondly, human infants stay with their mother for an exceptionally long period. It is common for mammals to remain with the mother until puberty and this occurs much later in humans (12 years compared with 34 months in farm animals). It is well known that the presence of the mother increase survival of the infants [3]. It follows that the absence of other mouths to feed increases the chances of children already born of surviving into adulthood. This survival of infants to puberty does impact directly on the chance for them to reproduce. Surprisingly, menopause does not correspond with the usual way nature chooses to maximise output. Indeed, in nature, quantity often supersedes quality. In humans, the 10-12 years investment to raise a child could justify the reduction in number.

In addition, the ability of grandmothers to help their daughters raise their own family is also beneficial to genetic lineage and is part of a selection process, although some authors support a more important mother effect than a grandmother effect [4].

Other arguments viewing menopause as a selective adaptation include the capacity of anovulatory women in a tribe to accumulate knowledge, a prerequisite to tribe fitness. For millennia, before literacy, knowledge resided in living brains and was exchanged verbally amongst living members of a community. This knowledge accumulation would not occur to the same extent with a shorter lifespan. Older members would make a tribe more knowledgeable and better equipped to deal with changes.

If menopause, by preventing reproduction in the latter part of a woman's life, creates a benefit for gene transmission, can an increasingly inefficient reproductive capacity also serve the same purpose? Although aneuploidy may result in disabled newborns, most of the time it results in no baby at all. In fact, most women experience a much reduced fertility at 40 , although menopause occurs much later. Could a second, separate mechanism have evolved in parallel to menopause in order to reduce late-age reproduction?

Aneuploidy could serve as a way for the ovary to reduce late reproduction or even multiple pregnancies when more than one egg is produced in a given cycle. If this were to be the case, then the production of aneuploidy oocytes would require the help of the uterus to discard dying embryos as a result of aneuploidy. Indeed we do not see a much greater frequency of aneuploidy at birth in humans than in animals.

A final point, exogenous ovarian stimulation with gonadotropins increases the rate of aneuploidy in several animals in which it has been studied [5, 6, 7], suggesting that the ovary could use chromosome segregation to prevent undesirable outcomes, such as multiple pregnancies in humans and cows, or uterine crowding in species like mouse and sheep. Although an absolute increase of aneuploidy has not been clearly demonstrated in humans treated with gonadotropins, the use of mild stimulation reduces its incidence [8]. In the older patient, there is an association between the endogenous level of gonadotropins and the risk of aneuploidy [9].

Could a mechanism that might have initially evolved to prevent multiple offspring in species where it is prevalent, have been recruited for limiting late-age reproduction?

There are still too many unanswered questions to fully support this hypothesis, but looking at the problem from this new angle may allow the development of a new research direction to improve oocyte quality in older women.

\section{References}

1. Ramesh MA, Malik SB, Logsdon Jr JM. A phylogenomic inventory of meiotic genes; evidence for sex in Giardia and an early eukaryotic origin of meiosis. Curr Biol. 2005;15(2):185-91.

2. Haadsma ML, Mooij TM, Groen H, Burger CW, Lambalk CB, Broekmans FJ, et al. A reduced size of the ovarian follicle pool is associated with an increased risk of a trisomic pregnancy in IVFtreated women. Hum Reprod. 2010;25(2):552-8.

3. Rashidi A, Shanley D. Evolution of the menopause: life histories andmechanisms. Menopause Int. 2009;15(1):26-30.

4. Peccei JS. A critique of the grandmother hypotheses: old and new. Am J Hum Biol. 2001;13(4):434-52.

5. Roberts R, Iatropoulou A, Ciantar D, Stark J, Becker DL, Franks S, Hardy K. Follicle-stimulating hormone affects metaphase 1chromosome alignment and increases aneuploidy in mouse oocytes matured in vitro. Biol Reprod. 2005;72(1):107-18

6. Ertzeid G, Storeng R. The impact of ovarian stimulation on implantation and fetal development in mice. Hum Reprod. 2001;16(2):221-5.

7. Veiga-Lopez A, Dominguez V, Souza CJ, Garcia-Garcia RM, Ariznavarreta C, Tresguerres JA, et al. Features of folliclestimulating hormone-stimulated follicles in a sheep model: keys to elucidate embryo failure in assisted reproductive technique cycles. Fertil Steril. 2008;89(5 Suppl):1328-37.

8. Baart EB, Martini E, Eijkemans MJ, Van Opstal D, Beckers NG, Verhoeff A, et al. Milder ovarian stimulation for in-vitro fertilization reduces aneuploidy in the human preimplantation embryo: a randomized controlled trial. Hum Reprod. 2007;22(4):980-8.

9. Dursun P, Gultekin M, Yuce K, Ayhan A. What is the underlying cause of aneuploidy associated with increasing maternal age? Is it associated with elevated levels of gonadotropins? Med Hypotheses. 2006;66(1):143-7. 